\title{
Metformin administration during pregnancy - current insight
}

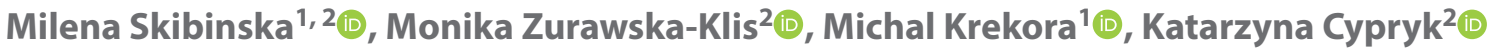 \\ ${ }^{1}$ Department of Obstetrics and Gynecology, Polish Mother's Memorial Hospital Research Institute, Lodz, Poland \\ 2Department of Internal Diseases and Diabetology, Central Clinical Hospital of The Medical University, Lodz, Poland
}

\begin{abstract}
The main mechanism of gestational diabetes mellitus (GDM) is insulin resistance, therefore using metformin as a medicine reducing insulin resistance appears to be promising.

Currently, the majority of medical associations do not recommend using metformin during pregnancy as the first-line of therapy when the diet regimen is insufficient for glycaemic control. However, they do allow its administration if there is no possibility of insulin treatment.

There is some evidence which suggests that using metformin during pregnancy is not related to an increased risk of obstetric complications during delivery and that its influence on the foetus can be beneficial.

Since metformin crosses the placenta, the major argument for cautious use of this drug are the potential long-term effects of the treatment for the child and its development in later life.

In this article, the authors attempt to discuss the use of metformin during pregnancy and the safety of the treatment in the light of current studies and recommendations.
\end{abstract}

Key words: metformin; pregnancy; diabetes mellitus; GDM

Ginekologia Polska 2021; 92, 1: 46-50

\section{INTRODUCTION}

The main cause of gestational diabetes mellitus (GDM) is increasing insulin resistance caused by a rise in the level of gestational hormones (progesterone, leptin, placental lactogen, cortisol) which are insulin antagonists. In some women there is no sufficient increase in insulin secretion (impaired first phase secretion) by the pancreas and hyperglycaemia may develop. The main factor determining insulinemia and the insulin tissue sensitivity in pregnant and non-pregnant patients is the body mass index (BMI). The higher the body weight, the lower the insulin sensitivity index. Also, the genetic (familiar diabetes occurrence; HLA DR 2, 3,4 mutation) and environmental factors (obesity, lack of exercise, excessive food intake) appear to be significant [1].

Metformin has been present in the treatment of diabetes for over sixty years but its potential application and therapeutic effects are not yet fully known. The initial evidence regarding the use of metformin in pregnant women came from 1975 reports of Aberdeen International Colloquia on sulphonylureas, biguanides and insulin in pregnancy. In developing countries, due to its low cost, metformin is commonly used now including during pregnancy.

Over the last sixty years, the importance of metformin in medicine has been evolving.

\section{THE MECHANISM OF ACTION OF METFORMIN}

The main effect of metformin therapy is a reduction of insulin-resistance by lowering the conversion of glycogen to glucose in the liver and enhancement of peripheral insulin sensitivity, especially in the muscles. Metformin also restricts intestinal absorption of glucose and regulates lipolysis and lipogenesis processes in the fatty tissue by lowering the levels of free fatty acids. Due to the suppressing mechanism of mTOR signalization and the mechanism related to enhancing the activity of tyrosine kinase (AMPK), metformin lowers the levels of glucose, insulin and inflammatory proteins in the blood, lowers the blood pressure and rate of metabolism, as well as suppresses angiogenesis and cell multiplication.

In 2017, Romero at al. postulated a potential preventive effect of metformin in preeclampsia. Romero's research suggests that the potentially by better the placental blood flow the lower the risk of preeclampsia $[2,3]$.

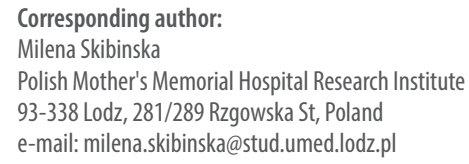


The main adverse effects of metformin include nausea, vomiting, abdominal pain and other gastrointestinal disorders. Metformin does not cause hypoglycaemia or weight gain [3].

Unfortunately, metformin crosses the placenta and its cord blood concentration is the same or even twice higher than that in the maternal blood. This gives rise to doubts concerning its use in everyday treatment during pregnancy [4].

\section{EFFECTIVENESS AND ACCEPTANCE OF METFORMIN USE IN PREGNANCY}

Metformin therapy seems to be attractive especially for the patients who wish to avoid insulin injections. Oral therapy is both simpler and more comfortable. A vast majority of pregnant women (about 77\%) treated with metformin stated that they would like the same treatment during their next pregnancy while only $28 \%$ of patients treated with insulin prefer it to metformin [5].

It should be stressed that there is no evidence of any spectacular medical effect during metformin therapy. In addition, in over $40 \%$ of pregnant women metformin therapy is insufficient for appropriate everyday glycaemic control and the inclusion of insulin is necessary, especially in patients with a higher BMl at early stages of pregnancy $\left(34 \mathrm{~kg} / \mathrm{m}^{2} \mathrm{vs}\right.$ $\left.31 \mathrm{~kg} / \mathrm{m}^{2}\right)$ and higher early blood glucose levels $(6.1 \mathrm{mmol} / \mathrm{L}$ vs $5.3 \mathrm{mmol} / \mathrm{L}$ ) [23]. Women diagnosed with GDM earlier, with higher BMI in early pregnancy and higher baseline glucose levels are more likely to require insulin and may be considered less suitable for oral medication [5].

Metformin is known for increasing the risk of lactic acidosis. In fact, concerns regarding the effects of another biguanide - phenformin - caused metformin to be withdrawn from the treatment of pregnant patients in many countries [6].

\section{METFORMIN IN WOMEN WITH POLYCYSTIC OVARY SYNDROME (PCOS), INSULIN RESISTANCE AND DIABETES MELLITUS TYPE 2 DURING PREGNANCY}

Nowadays, there seems to be a new epidemic not only of overweight and obesity, but also insulin resistance which may lead to a growing glucose intolerance and the resulting menstrual disorders and problems with becoming pregnant. As for the continuation of metformin therapy in women suffering from PCOS, insulin resistance, glucose intolerance or even diabetes type 2 who are at the beginning of pregnancy, there is still a limited number of studies regarding such patients. According to the ADA's 2016 recommendation metformin should be discontinued in those patients as soon as pregnancy is confirmed [6].

It should be also noted that hyperglycemia in early pregnancy, including women who suddenly discontinue metformin therapy, is teratogenic. Therefore, immediate insulin treatment should be introduced to ensure glycemia control. Moreover, metformin does not prevent GDM in women with PCOS [8].

Metformin therapy during pregnancy in women with pre-gestational diabetes mellitus type 2 is not well documented. It is likely that most women with type 2 diabetes before pregnancy will require treatment with insulin during their gestation to maintain glycaemic control.

In a retrospective study Hellmuth et al. [9] reported the use of metformin in 50 women, 19 of whom had type 2 diabetes. An increase in pre-eclampsia and perinatal mortality was noted.

In the group of pregnant women with diabetes mellitus type 2 or insulin-resistance present prior to pregnancy requiring greater insulin doses to ensure adequate glycemia control, the use of metformin as an insulin-saving therapy should be considered as potentially beneficial, although the issue warrants further research [5].

In the cohort from New Zealand, no increase in adverse pregnancy outcomes were observed in patients with type 2 diabetes mellitus [10].

Possibly, the safety of this therapy will be further determined pending the results of an ongoing randomized trial involving metformin treatment in diabetes type 2 during pregnancy [11].

\section{METFORMIN AND THE FIRST TRIMESTER CONCERNS}

There is no evidence of any potential teratogenic influence of the metformin resulting from its use during pregnancy [12].

The meta-analysis of studies in which 351 pregnant women with PCOS used metformin both before and during early pregnancy does not show an increased risk of serious congenital foetal defects [13].

In addition, there are some studies which confirm its higher efficacy in ovulation elicitation than clomiphene, which may prove significant given PCOS related fertility problems [14].

The observational study included 1250 women divided into two groups: metformin vs placebo. In the metformin group there were fewer lost pregnancies ( $8.8 \%$ vs $4.2 \%$ ), fewer miscarriages ( $26 \%$ vs $62 \%$ ), fewer cases of GDM ( $4 \%$ vs $26 \%)$, lower birth weight and lower incidence of pregnancy induced hypertension (PIH) [15].

\section{METFORMIN AND OBSTETRIC RISK}

There is some evidence that using metformin during pregnancy is unrelated with an increased risk of obstetric labour complications and that its influence on the foetus can be beneficial. 
In 2008, in a randomized clinical trial entitled Metformin in Gestational Diabetes (MiG) the authors postulated the following conclusions: among pregnant women treated with metformin there were fewer large for gestational age (LGA) infants, fewer cases of severe $(<1.6 \mathrm{mmol} / \mathrm{L})$ infant hypoglycaemia (reduction by about $60 \%$ ), the time spent in the intensive care unit was significantly shorter. In addition, metformin was linked to a reduction in women's weight gain during the whole observation period $(0.4 \pm 2.9 \mathrm{~kg}$ vs $2.0 \pm 3.3 \mathrm{~kg} ; \mathrm{p}<0.001)$. It is particularly noteworthy since lower weight gain during pregnancy is an independent factor reducing LGA incidence. The occurrence of preterm deliveries in this group was greater $(12.1 \%$ vs $7.6 \%, p=0.04)$ which was, however, not statistically significant [16].

A comparative study involving 1832 women with GDM who used either metformin or insulin revealed a lower weight gain in mothers and fewer LGA infants in the metformin group patients. At the same time, insulin was linked to a significantly higher risk of hypoglycaemia and PIH in mothers. There were no differences in maternal glycaemic control or obstetric outcomes [15].

Prospective and retrospective studies on a group of 857 pregnant women, where the effects of oral anti-diabetic agents (metformin, glibenclamide, acarbose, glyburide) and insulin were compared, revealed a higher rate of preeclampsia and perinatal infant deaths in the metformin group. In the oral anti-diabetic agents group, lower maternal weight gain and fewer cases of infant hypoglycaemia were observed. In the insulin group, there were more preterm deliveries and infants requiring observation at the intensive care unit (ICU). There were no differences in maternal glycaemic control or labour complications [15].

In the Farrar et al. [17] meta-analysis, two groups were analysed: the first one included patients treated with metformin and insulin added when necessary, while the other one was comprised of women treated only with insulin. The potential risk of LGA, macrosomia, admission to ICU and hypoglycaemia in infants as well as $\mathrm{PIH}$, preeclampsia and labour induction was lower in the metformin group. Instrumental birth and preterm birth were more frequent in the insulin group. Gestational age on delivery, birth weight and Apgar results did not differ between the groups.

\section{METFORMIN AND LONG-TERM EFFECTS ON CHILDREN}

After a 2-year observation of the infants born from mothers who took part in the 2008 MIG study, a follow-up project called Metformin in gestational diabetes: the offspring follow-up (MIG TOFU) was conducted. It showed that in the metformin group children had more subcutaneous fatty tissue but there was no difference in the amount of central fatty tissue, blood pressure or any other tested parameter in comparison to the children from the insulin group $[18,19]$.

In a follow-up study on the group of 7- to 9-year-old children of mothers involved in the MIG trial, the Adelaide Clinic subgroup (average age 7) showed no difference in body weight or structure (metformin vs insulin). By contrast, in the Auckland subgroup comprised of children whose average age was 9 , the metformin patients showed higher body mass, waist-hip ratio (WHR), arm and hip circumference [20].

In the other study, children whose mothers had taken metformin during pregnancy were heavier at 12 months of age and both heavier and taller at 18 months (12.0 vs $11.3 \mathrm{~kg}$ ). It should be underlined that only 96 children of mothers who suffered GDM were observed (31 treated with metformin only, 14 treated with metformin connected with insulin and 14 treated with insulin only) [21].

A systematic review and meta-analysis of Follow- up Studies of RCT's concerning long- term effects of oral antidiabetic drugs during pregnancy included 10 studies, with a maximal follow-up duration of 9 years, comprising 778 children of mothers with GDM or PCOS who were randomised to either metformin or insulin/placebo during pregnancy. Meta-analysis showed that children prenatally exposed to metformin were heavier compared to controls (standardised mean difference $=0.26,95 \% \mathrm{Cl} 0.11-0.41$ ), but not taller $(\mathrm{SMD}=0.10,95 \% \mathrm{Cl} 0.14-0.33)$. Additionally, offspring body mass index (BMI) $z$ scores did not differ according to metformin exposure (mean difference $=0.30,95 \% \mathrm{Cl} 0.01-0.61$ ). Individual small studies reported that prenatal exposure to metformin was associated with greater mid-upper arm, head and waist circumferences, biceps skinfolds, waist-to-height ratio, more arm fat, higher fasting glucose, ferritin and lower LDL cholesterol in offspring [22].

The latest 5-10 - year follow-up on children from the PregMet study, a double-blind, randomised controlled trial comparing metformin with placebo in PCOS pregnancies, examines the cardiometabolic risk factors in these children. Of the 255 invited children from the PregMet study, 141 (55\%) consented to participate. Maternal baseline characteristics in the first trimester were similar between the groups. Children in the metformin group had a higher BMI than those in the placebo group (mean difference $=0.41$, $95 \% \mathrm{Cl} 0.03-0.78, \mathrm{p}=0.03$ ). According to the authors an increased $\mathrm{BMI}$ in the metformin-exposed children might indicate a potential risk of inferior cardiometabolic health. Implications for adult health cannot be excluded [23].

Contrary to these facts, Butalia et al. [24] showed lower maternal weight gain, fewer LGA infants, fewer cases of pregnancy induced hypertension or events of intensive care unit infant hospitalization in the metformin group compared to the insulin alone group. There weres no difference in the incidence of preterm deliveries between the two groups. The 
psychomotor development in children of mothers with GDM at 2 years of age showed no difference compared to the other group.

In Wouldes et al. study comparing the influence of metformin and insulin on child development, the neurodevelopmental outcomes at 2 years of age were similar between the children born from mothers who were treated with metformin compared to those treated with insulin for GDM. Lower score on the standardized measures of neurodevelopment were predominantly associated with parental self-identified ethnicity, smoking in the household and clinical outcomes at birth unrelated to the treatment [25].

In New Zealand, one of the latest cohort studies into the effects of metformin vs insulin treatment of GDM which included 211 children followed up at 2 years, 128 were from Auckland, New Zealand (64 metformin vs 64 insulin), and 83 from Adelaide, Australia ( 39 metformin vs 49 insulin). No differences in the weight, height and psychomotor development of the children was noted [26].

\section{THE MEDICAL ASSOCIATIONS RECOMMENDATIONS}

The American Food and Drug Administration (FDA) places metformin in category $B$ of safety which means that animal reproduction studies did not demonstrate any risk to the foetus, however there are no corresponding well-documented studies on pregnant women [5].

According to the World Health Organisation (WHO) recommendation, metformin is acceptable but only as a second-line pharmacotherapy choice, after insulin. WHO currently recommends the use of metformin and glibenclamide in the resource limited areas where insulin administration involves logistic issues [27].

International Diabetes Federation (IDF) [2009] guidelines recommend the use of metformin in resource limited areas in patients who are least likely to comply with insulin. Metformin use is not recommended as the first-line of therapy when the diet is not efficient for glycaemic control before the 20 weeks of gestation and fasting glycemia exceeds $110 \mathrm{mg} / \mathrm{dL}$ or postprandial glycemia exceeds $140 \mathrm{mg} / \mathrm{dL}$ [28].

Both American College of Obstetricians and Gynecologists (ACOG) and American Diabetes Association (ADA) recommend insulin as the first-line medicine in gestational diabetes mellitus treatment when the diet regime is insufficient for glycaemic control. Metformin remains the second-line treatment [29].

Only the British National Institute for Health and Care Excellence (NICE) recommends metformin as a first-line therapy in women whose blood glucose levels do not stabilize after 1-2 weeks of diet and exercise regime. Insulin is recommended only in cases where metformin is contraindicated or unacceptable to the patient. At the same time, these recommendations indicate that the summary of product characteristics of metformin states that it should not be administered during pregnancy [30]. In the cases of prescribing metformin to treat GDM, the doctor is obliged to inform the pregnant women that the long-term effect of metformin on health or metabolism of the offspring during adulthood is still not known.

Conversely, the Society for Maternal-Fetal Medicine (SMFM) states that both insulin and metformin are safe and acceptable as the first-line treatment in GDM [31].

However, in 2018, Barbour LA et al. [32] published a cautionary response to the SMFM statement concerning pharmacological treatment of gestational diabetes. Even though the data on the short-term effects on mothers and foetuses appears to be promising, the potential long-term effects of metformin therapy — both beneficial and harmful — are still unknown. The authors emphasize the potential intrauterine programming and epigenetic aspects of the therapy. Metformin can suppress cell growth and mitochondrial respiration because in the later stages of pregnancy, when the number of mitochondrial transporters rises, metformin penetration into the mitochondria increases. In animal model research it has been demonstrated that metformin has negative effects on body weight and metabolism, which was pointed out in the 2018 publication.

International Federation of Gynaecology and Obstetrics (FIGO) recommends that insulin should be a first-line treatment also in developing countries in cases where one or more of the following occurs: diabetes mellitus is diagnosed before 20 weeks of gestation, diet regime is insufficient for glycaemic control during the period preceding 30 weeks of gestation, fasting blood glucose level exceeds $110 \mathrm{mg} / \mathrm{dL}$, postprandial blood glucose exceeds $140 \mathrm{mg} / \mathrm{dL}$ and body weight gain is over $12 \mathrm{~kg}$ [33].

Both Polish Diabetological Association and Polish Society of Gynaecologists and Obstetricians do not recommend any oral anti-diabetic agents (including metformin) in GDM treatment as these substances cross the placenta and there is no research on their long-term effects on the offspring. In women who use metformin it is recommended to begin insulin therapy as soon as pregnancy is confirmed [34, 35].

\section{CONCLUSIONS}

Metformin during pregnancy can be an alternative to insulin only when the patient refuses the therapy with insulin and it is obligatory to inform her about its potential negative influence on the foetus and later on the child.

There is no evidence of an increased risk of miscarriage or congenital foetal anomalies when using metformin.

Metformin reduces the maternal weight gain during pregnancy and the incidence of severe hypoglycaemia in infants. 
The major argument for cautious metformin administration are the still unknown or uncertain long-term effects of the treatment during pregnancy.

At present, the vast majority of medical associations does not recommend metformin during pregnancy as the first-line of therapy when the diet regimen is insufficient for glycaemic control and restrict its use to those cases in which there is no possibility of insulin treatment.

\section{REFERENCES}

1. Catalano PM, Kirwan JP, Haugel-de Mouzon S, et al. Gestational diabetes and insulin resistance: role in short- and long-term implications for mother and fetus. J Nutr. 2003; 133(5 Suppl 2): 1674S-1683S, doi: 10.1093/jn/133.5.1674S, indexed in Pubmed: 12730484.

2. Romero R, Erez O, Hüttemann M, et al. Metformin, the aspirin of the 21st century: its role in gestational diabetes mellitus, prevention of preeclampsia and cancer, and the promotion of longevity. Am J Obstet Gynecol. 2017; 217(3): 282-302, doi: 10.1016/j.ajog.2017.06.003, indexed in Pubmed: 28619690.

3. Garcia-Contreras C, Vazquez-Gomez M, Pesantez-Pacheco JL, et al. Maternal Metformin Treatment Improves Developmental and Metabolic Traits of IUGR Fetuses. Biomolecules. 2019; 9(5), doi: 10.3390/biom9050166, indexed in Pubmed: 31035702

4. Hague WM, Davoran PH, Mclntyre D, et al. Metformin crosses the placenta: a modulator for fetal insulin resistance. BMJ. 2003; 327: 880-881.

5. Lindsay RS, Loeken MR. Metformin use in pregnancy: promises and uncertainties. Diabetologia. 2017; 60(9): 1612-1619, doi: 10.1007/s00125017-4351-y, indexed in Pubmed: 28770325.

6. Salpeter S, Greyber E, Pasternak G, et al. Risk of fatal and nonfatal lactic acidosis with metformin use in type 2 diabetes mellitus. Cochrane Database Syst Rev. 2006(1): CD002967, doi: 10.1002/14651858.CD002967. pub2, indexed in Pubmed: 16437448.

7. American Diabetes Association. 14. Management of Diabetes in Pregnancy: . Diabetes Care. 2019; 42(Suppl 1): S165-S172, doi: 10.2337/dc19-S014, indexed in Pubmed: 30559240.

8. Vanky E, Stridsklev S, Heimstad R, et al. Metformin versus placebo from first trimester to delivery in polycystic ovary syndrome: a randomized, controlled multicenter study. J Clin Endocrinol Metab. 2010; 95(12): E448-E455, doi: 10.1210/jc.2010-0853, indexed in Pubmed: 20926533.

9. Hellmuth E, Damm P, Mølsted-Pedersen L. Oral hypoglycaemic agents in 118 diabetic pregnancies. Diabet Med. 2000; 17(7): 507-511, doi: 10.1046/j.1464-5491.2000.00314.x, indexed in Pubmed: 10972579.

10. Hughes RCE, Rowan JA. Pregnancy in women with Type 2 diabetes: who takes metformin and what is the outcome? Diabet Med. 2006; 23(3): 318-322, doi: 10.1111/j.1464-5491.2006.01750.x, indexed in Pubmed: 16492217.

11. Feig DS, Murphy K, Asztalos E, et al. MiTy Collaborative Group. Metformin in women with type 2 diabetes in pregnancy (MiTy): a multi-center randomized controlled trial. BMC Pregnancy Childbirth. 2016; 16(1): 173, doi: 10.1186/s12884-016-0954-4, indexed in Pubmed: 27435163.

12. Lee HY, Wei D, Loeken MR. Lack of metformin effect on mouse embryo AMPK activity: implications for metformin treatment during pregnancy. Diabetes Metab Res Rev. 2014; 30(1): 23-30, doi: 10.1002/dmrr.2451, indexed in Pubmed: 23983188.

13. Cassina $M$, Donà $M, D i$ Gianantonio $E$, et al. First-trimester exposure to metformin and risk of birth defects: a systematic review and meta-analysis. Hum Reprod Update. 2014; 20(5): 656-669, doi: 10.1093/humupd/dmu022, indexed in Pubmed: 24861556.

14. Wang R, Kim BV, van Wely M, et al. Treatment strategies for women with WHO group II anovulation: systematic review and network meta-analysis. BMJ. 2017; 356: j138, doi: 10.1136/bmj.j138, indexed in Pubmed: 28143834.

15. Singh AK, Singh R. Metformin in gestational diabetes: An emerging contender. Indian J Endocrinol Metab. 2015; 19(2): 236-244, doi: 10.4103/2230-8210.149317, indexed in Pubmed: 25729685.

16. Rowan JA, Hague WM, Gao W, et al. MiG Trial Investigators. Metformin versus insulin for the treatment of gestational diabetes. N Engl J Med. 2008; 358(19): 2003-2015, doi: 10.1056/NEJMoa0707193, indexed in Pubmed: 18463376.
17. Farrar $D$, Simmonds $M$, Bryant $M$, et al. Treatments for gestational diabetes: a systematic review and meta-analysis. BMJ Open. 2017; 7(6): e015557, doi: 10.1136/bmjopen-2016-015557, indexed in Pubmed: 28647726.

18. Rowan JA, Rush EC, Obolonkin V, et al. Metformin in gestational diabetes: the offspring follow-up (MiG TOFU): body composition at 2 years of age. Diabetes Care. 2011; 34(10): 2279-2284, doi: 10.2337/dc11-0660, indexed in Pubmed: 21949222.

19. Battin MR, Obolonkin V, Rush E, et al. Blood pressure measurement at two years in offspring of women randomized to a trial of metformin for GDM: follow up data from the MiG trial. BMC Pediatr. 2015; 15: 54, doi: 10.1186/s12887-015-0372-1, indexed in Pubmed: 25943394.

20. Rowan JA, Rush EC, Plank LD, et al. Metformin in gestational diabetes: the offspring follow-up (MiG TOFU): body composition and metabolic outcomes at 7-9 years of age. BMJ Open Diabetes Res Care. 2018; 6(1): e000456, doi: 10.1136/bmjdrc-2017-000456, indexed in Pubmed: 29682291.

21. ljäs $\mathrm{H}$, Vääräsmäki $M$, Saarela T, et al. A follow-up of a randomised study of metformin and insulin in gestational diabetes mellitus: growth and development of the children at the age of 18 months. BJOG. 2015; 122(7): 994-1000, doi: 10.1111/1471-0528.12964, indexed in Pubmed: 25039582.

22. van Weelden W, Wekker V, de Wit $L$, et al. Long-Term Effects of Oral Antidiabetic Drugs During Pregnancy on Offspring: A Systematic Review and Meta-analysis of Follow-up Studies of RCTs. Diabetes Ther. 2018; 9(5): 1811-1829, doi: 10.1007/s13300-018-0479-0, indexed in Pubmed: 30168045.

23. Hanem LG, Salvesen $\varnothing$, Juliusson PB, et al. Intrauterine metformin exposure and offspring cardiometabolic risk factors (PedMet study): a 5-10 year follow-up of the PregMet randomised controlled trial. Lancet Child Adolesc Health. 2019; 3(3): 166-174, doi: 10.1016/S2352-4642(18)303857, indexed in Pubmed: 30704873.

24. Butalia S, Gutierrez L, Lodha A, et al. Short- and long-term outcomes of metformin compared with insulin alone in pregnancy: a systematic review and meta-analysis. Diabet Med. 2017; 34(1): 27-36, doi: 10.1111/dme.13150, indexed in Pubmed: 27150509.

25. Wouldes TA, Battin M, Coat $S$, et al. Neurodevelopmental outcome at 2 years in offspring of women randomised to metformin or insulin treatment for gestational diabetes. Arch Dis Child Fetal Neonatal Ed. 2016; 101(6): F488-F493, doi: 10.1136/archdischild-2015-309602, indexed in Pubmed: 26912348.

26. Landi SN, Radke S, Engel SM, et al. Association of Long-term Child Growth and Developmental Outcomes With Metformin vs Insulin Treatment for Gestational Diabetes. JAMA Pediatr. 2019; 173(2): 160-168, doi: 10.1001/jamapediatrics.2018.4214, indexed in Pubmed: 30508164.

27. http://www.whqlibdoc.who.int/publications/2006/924159084X_eng. pdf?ua $=1$.

28. http://www.idf.org/webdata/docs/Pregnancy EN RTP.pdf.

29. Committee on Practice Bulletins-Obstetrics. ACOG Practice Bulletin No. 190: Gestational Diabetes Mellitus. Obstet Gynecol. 2018; 131(2): e49-e64, doi: 10.1097/AOG.0000000000002501, indexed in Pubmed: 29370047.

30. Diabetes in pregnancy: management from preconception to the postnatal period. NICE guideline 2015.

31. Society of Maternal-Fetal Medicine (SMFM) Publications Committee. Electronic address: pubs@smfm.org. SMFM Statement: Pharmacological treatment of gestational diabetes. Am J Obstet Gynecol. 2018; 218(5): B2-B4, doi: 10.1016/j.ajog.2018.01.041, indexed in Pubmed: 29409848.

32. Barbour LA, Scifres $C$, Valent $A M$, et al. A cautionary response to SMFM statement: pharmacological treatment of gestational diabetes. Am J Obstet Gynecol. 2018; 219(4): 367.e1-367.e7, doi: 10.1016/j. ajog.2018.06.013, indexed in Pubmed: 29959933.

33. Hod M, Kapur A, Sacks DA, et al. The International Federation of Gynecology and Obstetrics (FIGO) Initiative on gestational diabetes mellitus: A pragmatic guide for diagnosis, management, and care. Int J Gynaecol Obstet. 2015; 131 Suppl 3: S173-S211, doi: 10.1016/S00207292(15)30033-3, indexed in Pubmed: 29644654

34. Araszkiewicz A, Bandurska-Stankiewicz E, Budzyński A, et al. Zalecenia kliniczne dotyczące postępowania u chorych na cukrzycę 2019. Stanowisko Polskiego Towarzystwa Diabetologicznego. Diabeto IPrakt. 2019; 5(1): 65-68.

35. Wender-Ożegowska E, Bomba-Opoń D, Brązert J, et al. Standards of Polish Society of Gynecologists and Obstetricians in management of women with diabetes. Ginekol Pol. 2018; 89(6): 341-350, doi: 10.5603/GP.a2018.0059, indexed in Pubmed: 30010185 